\title{
Backscatter Measurements over Vegetation by Ground-Based Microwave Radars
}

\author{
Mehmet Kurum and Peggy O'Neill \\ NASA Goddard Space Flight Center, Hydrological Sciences Branch, \\ Code 614.3, Greenbelt, Maryland 20771, USA \\ Tel: (301) 614-6537, Fax: (301) 614-5808, email: Mehmet.Kurum@nasa.gov
}

\begin{abstract}
In the study of radar backscattering from vegetated terrain, it is important to understand how the electromagnetic wave interacts with vegetation and the underlying ground. In this paper, an expression of backscattering from a vegetation canopy in the case of spherical wave illumination is derived. Such an expression might apply to the practical case of a ground-based scatterometer overlooking vegetation. The relative importance of the beamwidth as well as the platform height on backscattering from vegetated terrain is studied. Preliminary results indicate that the discrepancy with plane wave illumination can be rather significant, and therefore should not be overlooked.
\end{abstract}

\section{Introduction}

Ground-based (tower- or truck-mounted) and aircraft-based sensors at L-band frequency have played an important role in research of remote sensing of soil moisture for more than thirty years [1]. The Mmicrowave data collected in field campaigns have beenwere very valuable for understanding and modeling of microwave interactions with soil and vegetation cover, for conducting sensitivity analyseis of scene and instrument parameters, and most importantly, for evaluating performance of viable retrieval algorithms over a range of spatial scales from field to satellite footprint scale [2, 3]. Due to several L-band space missions, most notably ESA's Soil Moisture Ocean Salinity (SMOS) mission (launched November 2009) and NASA's Soil Moisture Active Passive (SMAP) mission (to be launched 2014/2015), ground- and aircraft-based microwave instruments are centrally placed within the heart of the calibration/validation activities to ensure that high quality data products are generated and archived for science purposes $[4,5]$. Over the longer term, these measurements will assist in assessing the performance of the spaceborne soil moisture retrieval algorithms in the presence of vegetation, topography, temporal change, heterogeneity, and other factors.

In active microwave remote sensing of soil moisture through vegetation, analyseis of the backscatter data acquired by ground-based radars were usually carried out by microwave discrete vegetation models based on analytical wave formulation [6-9] or phenomenological radiative transport theory [10-13]. The uniform plane wave assumption for incident and scattered waves is often introduced in these models due mainly to simplicity, and the scattered power is usually represented in terms of backscattering coefficient, which is proportional to the ratio of far-field observation of backscattered power to incident power of plane wave illumination. With this assumption, the spherical wave phase front is approximated as a planar one over the area illuminated by the radar, thus facilitating the scattering coefficient computations. However, iIn practice, especially in those situations where ground-based scatterometers are employed, the plane wave approximation is, however, violated and the illumination over the target is non-uniform. The measured scattering coefficient by ground-based scatterometers is distorted by antenna illumination which depends on antenna beamwidth, operating frequency, incidence angle, and radar altitude relative to vegetation depth.

To investigate the antenna illumination effects such as radar altitude on the measurements of the backscattering coefficient, the mechanisms of microwave scattering from a vegetation canopy should be considered in the presence of actual antenna radiation. In this study, the radar characteristics areis directly incorporated into analytical wave theory in conjunction with the distorted Born approximation (DBA), a first order scattering approximation [6]. Unlike previous studies in which plane wave illumination is assumed, the antenna radiation is replaced with itstheir plane wave spectrum representation. The incident plane waves of differing propagation vectors travel through the mean medium [14] and are scattered by the particles embedded in this medium. DBA is used to describe the vegetation scattering process of each ray of the spatial spectrum of the scattered field, which is associated with statistical characteristics of individual scatterers in the vegetation layer. The received signal is expressed as a weighted coherent sum involving the antenna spatial spectrum and backscattered radiation. This analytical wave formulation accounts for the spread of the beam within the medium and for the variation of its intensity and phase with the propagation direction. 
The backscatter data used in this study are provided by a truck-based radar/radiometer system called ComRAD (for Combined Radar/Radiometer) at a fixed altitude and multiple incidence angles. The microwave data along with a variety of ground truth data were collected over stands of small planted deciduous small Paulownia trees with broad leaves in 2006-2007 [17] and over a natural stand of small Virginia pine coniferous trees in 2008-2009 [18]. In this paper, the results from these experiments will be briefly described and the data will be discussed from the perspective of the platform height effects such as beam divergence, spreading loss, and radiation characteristics of the antenna.

\section{Calculation of Radar Scattering Coefficients}

In this paper, we start by examining a bistatic scattering from a forested terrain as illustrated in Fig. 1, where the forest is represented as an ensemble of canonical scatterers located above a flat ground with a diffuse upper boundary and two antennas (receive and transmit) are overlooking the forest. It is assumed that the canopy consists of randomly distributed branches and leaves, and vertically distributed tree trunks all having prescribed location and orientation statistics. Branches and trunks are modeled as finitely long, circular, lossy-dielectric cylinders, and leaves are modeled as at thin dielectric discs. These canonical scatterers are assumed to be uniformly distributed throughout the layer. By the Foldy theory [14], the forest layer is replaced by a slab of equivalent continuous (mean) medium. The transmit and receive antennas with arbitrary radiation patterns areis directly incorporated into analytical wave theory in conjunction with the distorted Born approximation (DBA) [6]. An expression for the received field due to a single particle immersed in a slab of mean medium over a flat ground surface is derived. This formulation assumes no knowledge of the incident field on the random medium, and therefore holds for any arbitrary wave form. It takes explicit account of the transmit and receive antenna radiation properties along the wave directions.

In order to determine the field at the receiver, the scattered fields from the illuminated medium need to be calculated. The plane wave spectrum of the scattered fields arises as a consequence of all incident plane waves of differing propagation vectors being scattered by the medium into the direction of antenna. The scattered plane wave spectrum is computed by employing the DBA such that the incident and scattered waves propagate with the characteristics of the mean wave. By means of the reciprocity relation [15], the receiver response to the scattered field is obtained as an integral over the angular coordinates involving the plane wave spectrum of the scattered fields and the radiation field of the receiving antenna. This expression represents a general form for the received field due to a single scatterer immersed in the mean medium and illuminated by an arbitrary incident wave. It is important to note that this formulation does not require that the scatterers (receiver antenna) be positioned in the far field of the receive antenna (scatterers). It consists of two double integrals, where one is with respect to the transverse wave number of incident waves and the other one is with respect to the transverse wave number of scattered waves. These integrals are immediately dispensed with via a joint 4-dimensional asymptotic stationary phase evaluation [16] under the assumption that the regular far-field conditions hold: ; (1) each scatterer in the vegetation layer is in the far-zone of the antennas,; and (2) the antennas are in the far-zone of each scatterer within the layer. The condition that the antenna is in the farfield of the medium depth is, however, not met. This allows us to use this general received expression to determine what amount the far-field scattering coefficients lacks with respect to vegetation depth. Since the most of the measurement techniques requires only that backscattered fields be determined, further discussion is restricted to the monostatic case. Upon specializing the backscatter antenna configuration (see Fig. 2), the antenna is situated in the farzone of the depth of the layer in addition to the far-field conditions mentioned above. With this assumption, the backscattering coefficient reduces to the form derivable in the case of a plane wave incidence [7].

A coherent scattering algorithm based on a Monte Carlo simulation is developed to demonstrate the effect of antenna characteristics on the radar response of distributed scatterers. The aforementioned formulation is extended to a multi-layer forest canopy with an underlying rough surface to make it more realistic. In the simulations, the scattered fields are computed by coherently adding the scattered field from each scatterer randomly positioned in the illuminated volume. Due to the spread of the wave, different particles in the layer experience different incidence directions. The coherent sum of these scattered waves results in averaging the scattering mechanisms' strength over the illuminated volume. The preliminary simulation results indicate that divergence of beam results in the weaker scattered fields in the bistatic direction associated with canopy-ground interaction since the scattered wave from vertical trunks becomes slightly away from the forward scattering cone, and scattered and incident waves are no longer parallel to each other as shown in Fig. 2.

\section{Conclusion}


In the analysis of microwave ground-based data, various theoretical vegetation models have been used with the assumption of plane wave incidence. These models and usually overlooked radarthe platform height effects such as beam divergence, spreading loss, and radiation characteristics of the antenna. The exact range of applicability for the plane wave assumption for geometries of ground-based systems, however, quite ambiguous since many other parameters s such as antenna beamwidth, radar altitude, and vegetation depth may also be important factors in the radar backscattering process. This study examines the antenna illumination effects such as platform height on the measurements of the backscattering coefficient by a ground-based scatterometer. Although this study is still in progress, preliminary results indicate that the antenna pattern and sphericity of the wavefront must be taken into account in the case of remote sensing of distributed targets, i.e. vegetated terrains, by ground-based sensors.

\section{References}

1. P. E. O'Neill, T. J. Jackson, D. Entekhabi, E. Njoku, "Survey of L Band tower and airborne sensor systems relevant to upcoming soil moisture missions,” IEEE Geoscience and Remote Sensing Newsletter, 151:13-16, 2009

2. T. Schmugge, P. E. O'Neill, and J. R. Wang, "Passive microwave soil moisture research”, IEEE Transactions on Geoscience and Remote Sensing, vol. GE-24, no. 1, pp 12-22, Jan 1986

3. M. C. Dobson and F. T. Ulaby, “Active microwave soil moisture research”, IEEE Transactions on Geoscience and Remote Sensing, vol. GE-24, no. 1, pp 23-36, Jan 1986

4. Y. H. Kerr, P. Waldteufel, J. P. Wigneron, S. Delwart, et. al., "The SMOS Mission: New Tool for Monitoring Key Elements of the Global Water Cycle,” Proceedings of the IEEE, vol. 98, no. 5, pp. 666 - 687, May 2010.

5. D. Entekhabi, E. Njoku, P. E. O’Neill, K. Kellogg, et. al., "The Soil Moisture Active and Passive (SMAP) Mission,” Proceedings of the IEEE, vol. 98, no. 5, pp. 704 - 716, May 2010.

6. R. H. Lang, "Electromagnetic backscattering from a sparse distribution of lossy dielectric scatterers," Radio Science, vol. 16, no. 1, pp. 15-30, 1981.

7. N. S. Chauhan, R. Lang, and K. J. Ranson, "Radar modeling of a borel forest,” IEEE Transactions on Geoscience and Remote Sensing, vol. 29, no. 4, pp. 627-638, 1991.

8. R. H. Lang, R. Landry, O. Kavaklioglu, and J. C. Deguise, "Simulation of microwave backscatter from a red pine stand,” in Proc. SPIE, Multispectral Microw. Sens. Forestry, Hydrol. Natural Resources, Rome, Italy, vol. 2314, pp. 538-548, 1994

9. Y.-C. Lin and K. Sarabandi, "A Monte Carlo coherent scattering model for forest canopies using fractal-generated trees,” IEEE Transactions on Geoscience and Remote Sensing, vol. 37, pp. 440-451, Jan. 1999

10. F. T. Ulaby, K. Sarabandi, K. McDonald, and M. C. Dobson, "Michigan microwave canopy scattering model," International Journal of Remote Sensing, vol. 11, pp. 1223-1253, 1990.

11. P. Ferrazoli, D. Solimini, G. Luzi, and S. Palocia, "Model analysis of backscatter and emission from layered vegetation,” J. Electromagn. Waves Appl., vol. 25, pp. 175-193, 1991.

12. M. A. Karam, A. K. Fung, R. H. Lang, N. S. Chauhan, , “A microwave scattering model for layered vegetation ," IEEE Transactions on Geoscience and Remote Sensing, vol. 30, no. 4, pp 767 - 784, Jul 1992.

13. L. Tsang, C. H. Chan, J. A. Kong, and J. Joseph, "Polarimetric signature of a canopy of dielectric cylinders based on first and second order vector radiative transfer theory,” J. Electromagn. Waves Applicat., vol. 6, pp.19-51, 1992.

14. L. Foldy, “The multiple scattering of waves,” Phys. Rev., vol. 67, no. 3, pp. 107-119, 1945.

15. W. Wasylkiwskyj, "Response of an antenna to arbitrary incident fields," in Proceedings of Antennas and Propagation Society International Symposium, vol. 3B, pp. 39-42, Washington, DC, USA, Jul. 2005. 
16. R.

Collin

F.
Transmit
Antenna

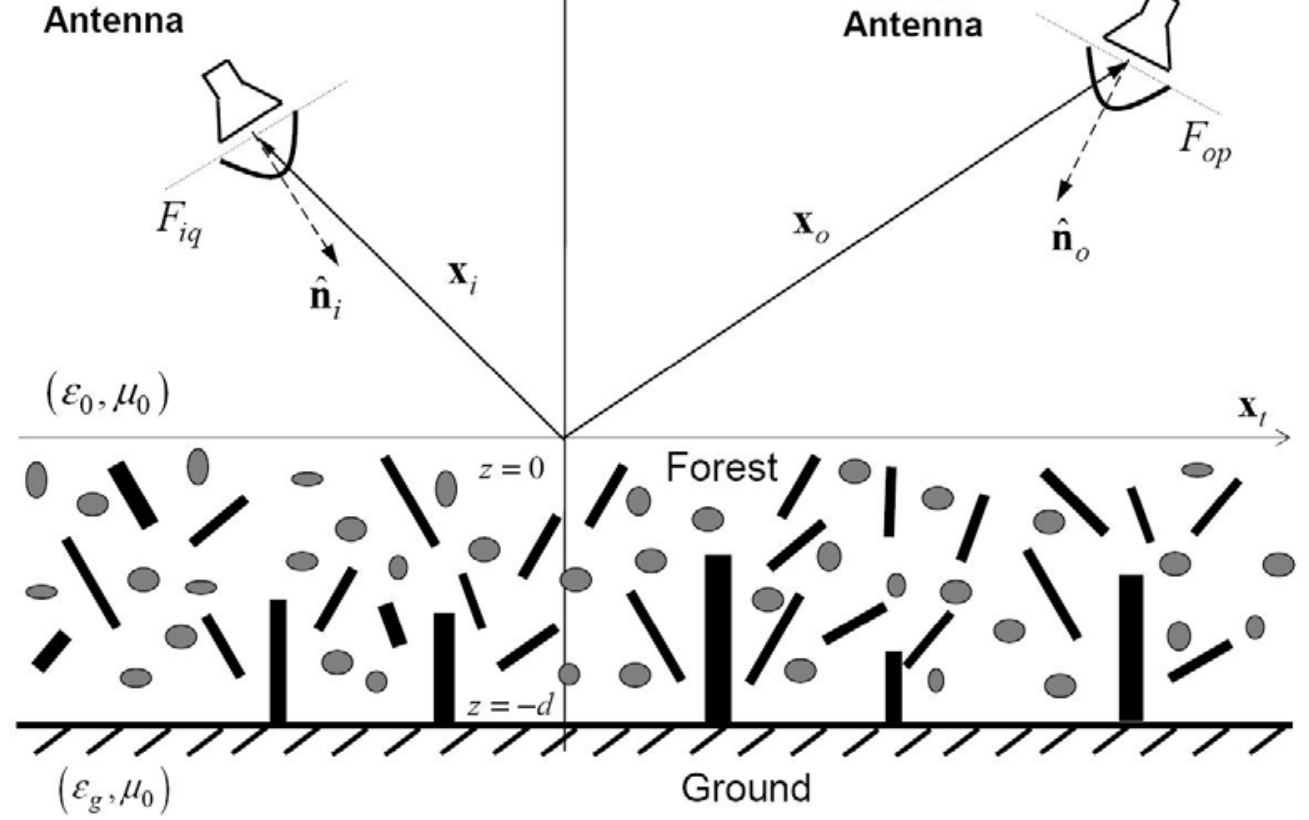

and $\mathrm{J}$.

E.

Figure 1 Bistatic configuration over a forest canopy

Zucker, Antenna Theory, vol. 1, ch. 3. McGraw Hill, 1969. 


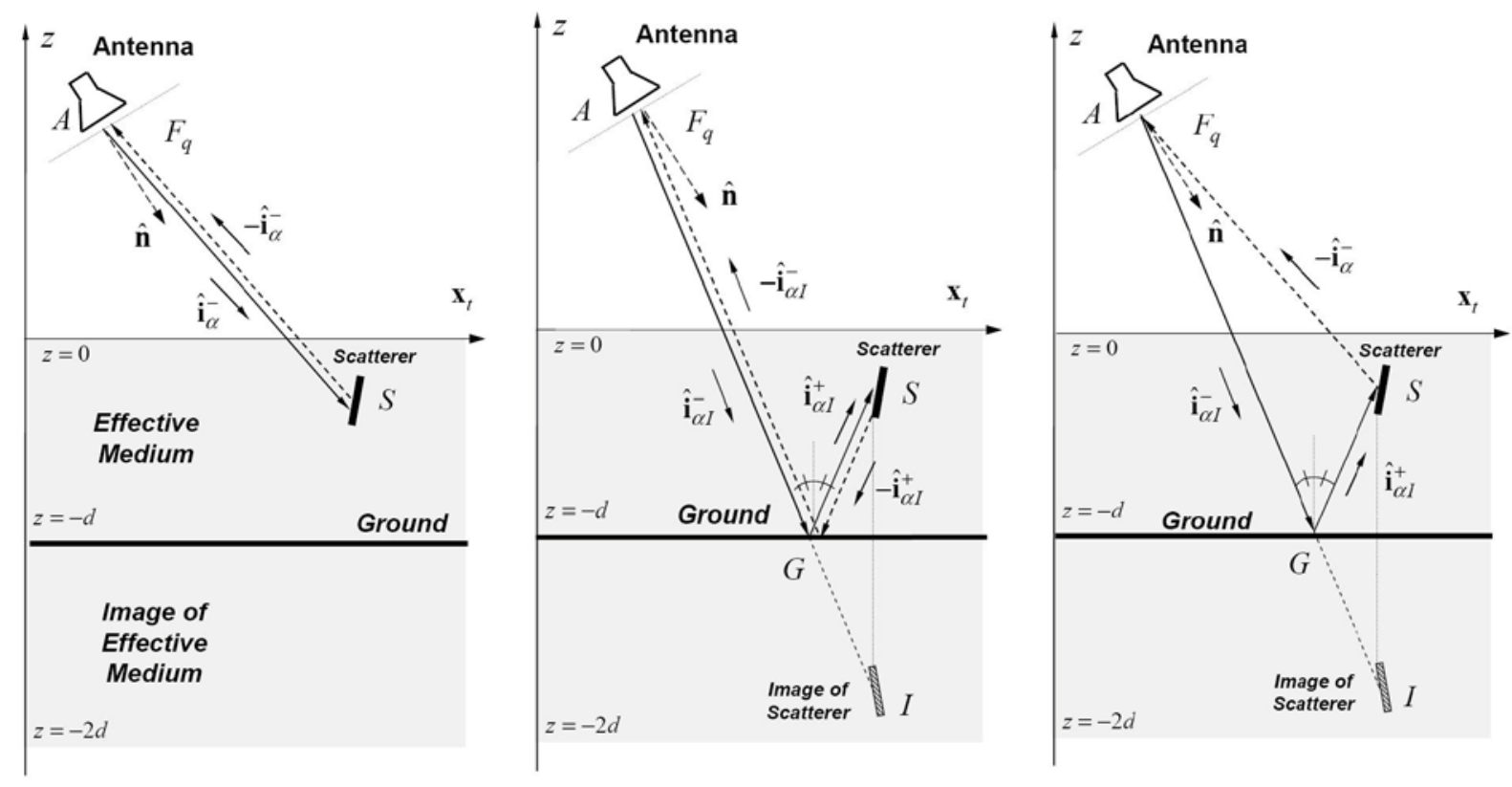

Figure 2 Backscattering mechanisms: Direct, direct-reflected, and reflected terms from left to right, respectively. 